\title{
Liposomes as carriers of poorly water-soluble substrates: linear modelling of membrane systems with catalytic or binding sites of different facedness
}

\author{
Significance of experimental membrane partition coefficients and of kinetic and equilibrium \\ parameters
}

\author{
Karel P. M. HEIRWEGH, ${ }^{*} \ddagger$ Jules A. T. P. MEUWISSEN,* Marc VERMEIR* and Humbert DE SMEDT $\dagger$ \\ ${ }^{*}$ Laboratory of Hepatology and †Laboratory of Physiology, Faculty of Medicine, Katholieke Universiteit Leuven, Gasthuisberg, \\ B-3000 Leuven, Belgium
}

\begin{abstract}
1. A multiphasic modelling approach to systems containing membrane-bound receptors or catalytic sites and a liposomal preparation as a substrate carrier is described. Kinetic expressions are derived for a singlesubstrate enzymic reaction operating at constant liposome concentration or at a fixed substrate/liposome concentration ratio. 2 . The assumption that accumulation of exchangeable components into the phospholipid bilayers can be described by linear bulk-phase partition leads to simple relationships between the initial reaction rate and (a) two kinetic coefficients $\left(V\right.$ and $\left.K_{\mathrm{m}}^{\prime}\right)$, (b) the partition coefficients of the solutes for the lipid compartments of the membrane $\left(P_{\mathrm{s}}^{\mathrm{m}}\right)$ and liposomal preparations $\left(P_{\mathrm{s}}^{\mathrm{l}}\right)$ and $(\mathrm{c})$ the total concentrations of substrate, membrane lipid and liposomal lipid. $K_{\mathrm{m}}^{\prime}$ is called the effective Michaelis constant. 3. For correct estimation of the coefficients $V, K^{\prime}, P_{\mathrm{s}}^{\mathrm{m}}$ and $P_{\mathrm{s}}^{\mathrm{l}}$ extrapolation to zero lipid concentration is required. 4 . The distinction is introduced between hydrophilic and hydrophobic aqueous-faced sites, lipid-faced sites and mixed sites, i.e. sites overlapping an aqueous and a lipid region. For hydrophilic aqueous-faced sites $K_{\mathrm{m}}^{\prime}$ is equal to the true $K_{\mathrm{m}}$ and for the other types of site to $K_{\mathrm{m}} / P_{\mathrm{s}}$. For lipid-faced and for mixed sites $P_{\mathrm{s}}$ corresponds to the membrane partition coefficient $P_{\mathrm{s}}^{\mathrm{m}}$. For binding of homologous compounds to a hydrophobic aqueous-faced binding pocket $P_{\mathrm{s}}$ is the incremental site partition coefficient $P_{\mathrm{s}}^{\mathrm{bs}}$, which takes into account the energetic contribution to the binding process due to the hydrophobic tail of the ligands. 5 . $K_{\mathrm{m}}^{\prime}$ accounts for any effects due to the facedness and nature of the enzymic sites. The dependence of the systems on the size of the lipidic partition compartment(s) is expressed exclusively by a distribution function $F .6$. When enzyme assays are performed with a series of chemically different substrates containing the same catalytically sensitive group, independence of $K_{\mathrm{m}}^{\prime}$ from partition indicates a hydrophilic aqueous-faced binding site. For the low-molecular-mass members of the homologous series a linear increase in - $\log$ $\left(K_{m}^{\prime}\right)$ with the logarithm of the partition coefficient will be observed with any of the other site types considered 7. Equilibrium relationships for binding of a ligand to a membrane-bound receptor are also derived. 8. The significance of experimental membrane partition coefficients is discussed.
\end{abstract}

\section{INTRODUCTION}

Reactions involving membrane-bound receptors and enzyme systems are usually described by the mathematical formalism developed for homogeneous systems, leading to ill-defined equilibrium or kinetic parameters and to loss of potentially interesting information. In some theories, the multiphasic nature of the systems has been taken into account by postulating ligand binding to phospholipid binding sites (see, e.g. Tipping et al., 1979b) or partition into aqueous and lipidic fluid phases of defined volume (Cho \& Miwa, 1973; Parry et al., 1976; Heirwegh et al., 1978).

In the present paper, the local concentrations are expressed in terms of mole fractions (Hill, 1974; Ragan, 1978). By further postulating linear solute partition in bilayer membranes, simple and fairly generally applicable procedures can be developed for modelling biochemical reactions occurring in multiphasic systems. Apart from aqueous-faced and lipid-faced types of binding site, as defined by Parry et al. (1976), it was found useful to distinguish aqueous-faced sites further with hydrophobic binding patches and mixed sites overlapping part of an aqueous and a lipidic compartment. The approach is illustrated in some detail for membrane-bound enzymes acting in the presence of a liposomal preparation as a substrate carrier, since this procedure (Noy \& Zakim, 1985; Whitmer et al., 1986) may offer a valuable alternative to the use of a detergent or co-solvent (Hook et al., 1973) or of a binding protein (Bartlett et al., 1985). In the former approach it may not be possible to formulate quantitatively all the chemical reactions occurring in the system, whereas in the latter non-linear binding considerably complicates modelling and parameter estimation. The significance of experimental membrane partition coefficients and of the kinetic or equilibrium parameters that can be derived from the experimental data is discussed.

$\ddagger$ To whom correspondence and reprint requests should be sent.

Vol. 254 


\section{THEORY}

In aqueous suspensions of liposomal material and a membrane-bound enzyme or receptor, the solutes can partition into the following compartments: the lipid phases of the membrane and liposomal preparation, and the corresponding lumenal and surrounding aqueous phases. We begin by deriving rate equations for some relatively simple kinetic systems, containing either aqueous-faced hydrophilic (aq) or lipid-faced (m) binding sites. At a later stage two additional types of binding site will be considered.

On the assumption that the local concentrations in the lumenal and surrounding aqueous compartments are identical for any species intervening in the chemical reaction(s) occurring in the system, one can consider the aqueous compartments as a single aqueous phase. Symbols relating to substrates, products and other chemical species are provided by the superscript $i$, which is $\mathrm{m}, \mathrm{l}$ or aq for species present in the membrane lipid, liposomal lipid or aqueous phase, respectively.

Suppose that a membrane-bound enzyme, with the enzymic sites located in phase $i$, catalyses the reversible reaction:

$$
\mathrm{E}^{\mathrm{i}}+\mathrm{S}^{\mathrm{i}} \rightleftharpoons(\mathrm{ES})^{\mathrm{i}} \rightleftharpoons(\mathrm{EP})^{\mathrm{i}} \rightleftharpoons \mathrm{E}^{\mathrm{i}}+\mathrm{P}^{\mathrm{i}}
$$

with $\mathrm{i}=\mathrm{m}$ for membrane-faced enzymic sites and $\mathrm{i}=\mathrm{aq}$ for hydrophilic aqueous-faced sites. $S^{i}$ and $P^{i}$ are the substrate and product, and $E^{i},(E S)^{i}$ and $(E P)^{i}$ free enzyme, enzyme-substrate and enzyme-product complex, respectively. In the absence of product, the initial reaction rate $v^{\mathrm{i}}$ is given by:

$$
v^{\mathrm{i}}=\frac{\mathrm{d} x_{\mathrm{p}}^{\mathrm{i}}}{\mathrm{d} t}=k \cdot x_{\mathrm{E}}^{\mathrm{i}} \frac{x_{\mathrm{s}}^{\mathrm{i}}}{K_{\mathrm{m}}+x_{\mathrm{s}}^{\mathrm{i}}} \text { with } x_{\mathrm{E}}^{\mathrm{i}}=x_{\mathrm{e}}^{\mathrm{i}}+x_{\mathrm{es}}^{\mathrm{i}}
$$

where $k$ is a proportionality factor (Reiner, 1969) and $K_{\mathrm{m}}$ the Michaelis constant. The local concentrations in phase $\mathrm{i}$ of substrate and product are $x_{\mathrm{s}}^{\mathrm{i}}$ and $x_{\mathrm{p}}^{\mathrm{i}}$, and $x_{\mathrm{E}}^{\mathrm{i}}$, $x_{\mathrm{e}}^{\mathrm{i}}$ and $x_{\mathrm{es}}^{\mathrm{i}}$ are those of total, free and occupied enzymic sites, respectively. Local concentrations $x_{j}^{i}$ are expressed in terms of mole fractions:

$$
x_{\mathrm{j}}^{\mathrm{i}}=n_{\mathrm{j}}^{\mathrm{i}} / N^{\mathrm{i}}
$$

where $n_{j}^{1}$ is the number of moles of component $J$ present in phase $i$ and $N^{i}$ the total amount of substance in moles, or the chemical content, of phase $i$.

To render the analysis generally useful, the reaction rate has to be formulated as a function of the total substrate concentration. The relationship between the local concentration of substrate $x_{s}^{i}$ and its total concentration is determined by the chemical and physical processes the substrate undergoes. These include binding of substrate to the enzymic site and conversion to product, and partition in both the membrane and liposome preparations. The reactions or steps of mass transport linking the exchangeable chemical species $S^{\mathbf{1}}$ and $\mathrm{P}^{i}$ present in the various compartments are assumed to be reversible and rapid compared with enzymic conversion of the substrate. This permits the local concentration $x_{\mathrm{s}}^{1}$ to be calculated from the set of nonenzymic reactions as if they were at equilibrium in the system, which, as a whole, is thus in a state of partial equilibrium.

Assuming linear exchange reactions between homogeneous phases:

$$
\mathrm{S}^{\mathrm{aq}} \rightleftharpoons \mathrm{S}^{\mathrm{m}} \text { and } \mathrm{S}^{\mathrm{aq}} \rightleftharpoons \mathrm{S}^{1}
$$

one can describe the partition process by bulk-phase partition coefficients $P_{\mathrm{s}}^{\mathrm{m}}$ for the membrane phase and $P_{\mathrm{s}}^{\mathrm{l}}$ for the liposome phase:

$$
P_{\mathrm{s}}^{\mathrm{m}}=x_{\mathrm{s}}^{\mathrm{m}} / x_{\mathrm{s}}^{\mathrm{aq}} \text { and } P_{\mathrm{s}}^{\mathrm{l}}=x_{\mathrm{s}}^{\mathrm{l}} / x_{\mathrm{s}}^{\mathrm{aq}}
$$

Neglecting enzyme-bound substrate the mass balance on substrate is:

$$
n_{\mathrm{s}}=n_{\mathrm{s}}^{\mathrm{aq}}+n_{\mathrm{s}}^{\mathrm{m}}+n_{\mathrm{s}}^{\mathrm{l}}
$$

where $n_{\mathrm{s}}$ is the total amount of substrate in the system, and $n_{\mathrm{s}}^{\mathrm{aq}}, n_{\mathrm{s}}^{\mathrm{m}}$ and $n_{\mathrm{s}}^{\mathrm{l}}$ are the amounts present in the aqueous phase, membrane phase and liposome phase, respectively. Taking into account the definition of the mole fraction (3) and the partition relationships (5), the amounts of substrate present in the membrane $\left(n_{\mathrm{s}}^{\mathrm{m}}\right)$ and liposomal phase $\left(n_{\mathrm{s}}^{1}\right)$ are given by:

$$
n_{\mathrm{s}}^{\mathrm{m}}=P_{\mathrm{s}}^{\mathrm{m}} \cdot x_{\mathrm{s}}^{\mathrm{aq}} \cdot N^{\mathrm{m}} \text { and } n_{\mathrm{s}}^{1}=P_{\mathrm{s}}^{\mathrm{l}} \cdot x_{\mathrm{s}}^{\mathrm{aq}} \cdot N^{1}
$$

Introduction into eqn. (6) yields:

$$
n_{\mathrm{s}}=n_{\mathrm{s}}^{\mathrm{aq}}+x_{\mathrm{s}}^{\mathrm{aq}} \cdot P_{\mathrm{s}}^{\mathrm{m}} \cdot N^{\mathrm{m}}+x_{\mathrm{s}}^{\mathrm{aq}} \cdot P_{\mathrm{s}}^{\mathrm{l}} \cdot N^{\mathrm{l}}
$$

and with $n_{\mathrm{S}} / N^{\mathrm{aq}}$ as a measure of the total substrate concentration $S$ :

$$
S=n_{\mathrm{s}} / N^{\mathrm{aq}}=x_{\mathrm{s}}^{\mathrm{aq}} \cdot\left[1+P_{\mathrm{s}}^{\mathrm{m}}\left(N^{\mathrm{m}} / N^{\mathrm{aq}}\right)+P_{\mathrm{s}}^{\mathrm{l}}\left(N^{\mathrm{l}} / N^{\mathrm{aq}}\right)\right]
$$

The total concentrations of enzyme $(E)$, product $(P)$, membrane lipid $(M)$ and liposomal lipid $(L)$ are defined in an analogous way:

$$
E=n_{\mathrm{E}} / N^{\mathrm{aq}}, P=n_{\mathrm{P}} / N^{\mathrm{aq}}, M=n_{\mathrm{M}} / N^{\mathrm{aq}}, L=n_{\mathrm{L}} / N^{\mathrm{aq}}
$$

where $n_{\mathrm{E}}, n_{\mathrm{P}}, n_{\mathrm{M}}$ and $n_{\mathrm{L}}$ are the total amounts of enzymic sites, product, membrane and liposomal lipid present in the system, respectively. Now assume that the lipidic solutions are sufficiently dilute to satisfy the conditions:

$$
\sum_{\mathrm{j}} n_{\mathrm{j}}^{\mathrm{m}} \ll n_{\mathrm{M}} \text { and } \sum_{\mathrm{j}} n_{\mathrm{j}}^{1} \ll n_{\mathrm{L}}
$$

where the summations extend over the solutes present in lipid phase $\mathrm{m}$ or 1 . Thus, replacing in eqn. (9) the chemical contents $N^{\mathrm{m}}$ and $N^{1}$ by the amounts of lipid $n_{\mathrm{M}}$ and $n_{\mathrm{L}}$, respectively, one obtains:

$$
S=x_{\mathrm{s}}^{\mathrm{aq}} \cdot\left(1+P_{\mathrm{s}}^{\mathrm{m}} \cdot M+P_{\mathrm{s}}^{\mathrm{l}} \cdot L\right)
$$

This equation is valid insofar as (a) the linear-partition hypothesis (5) and the mass balance equation (6) are verified and (b) the lipidic solutions are sufficiently dilute to satisfy the inequalities (11). The latter condition may be less restrictive than would appear on first inspection since constant membrane partition coefficients were found for testosterone and some other neutral amphiphiles up to ligand/phospholipid ratios of about 0.1 (Tipping et al., 1979a).

The function $F_{s}$, which is called the 'distribution function' of the substrate:

$$
F_{\mathrm{s}}=S / x_{\mathrm{s}}^{\mathrm{aq}}=1+P_{\mathrm{s}}^{\mathrm{m}} \cdot M+P_{\mathrm{s}}^{\mathrm{l}} \cdot L
$$

establishes the relationship between the concentration in the aqueous phase $x_{\mathrm{s}}^{\mathrm{aq}}$ and the total concentration $S$. A similar distribution function $F_{j}$ can be defined for each exchangeable solute $\mathrm{J}$.

The enzymic reaction rate can now be expressed in terms of total concentrations. To the extent that the amounts of substrate and product present in the phase 
containing the enzymic sites can be neglected compared with the total amount of all other components:

$$
\left(n_{\mathrm{s}}^{\mathrm{i}}+n_{\mathrm{p}}^{\mathrm{i}}\right) \ll \sum_{k} n_{k}^{\mathrm{i}}
$$

it follows that $\mathrm{d} x_{\mathrm{p}}^{\mathrm{i}} / \mathrm{d} t=\left(\mathrm{d} n_{\mathrm{p}}^{\mathrm{i}} / \mathrm{d} t\right) / N^{\mathrm{i}}$. A measure of the initial reaction rate $v$ for the system as a whole is then given by:

$$
v=\frac{\mathrm{d} P}{\mathrm{~d} t}=k \cdot E \frac{x_{\mathrm{s}}^{\mathrm{i}}}{K_{\mathrm{m}}+x_{\mathrm{s}}^{\mathrm{i}}}=V \frac{x_{\mathrm{s}}^{\mathrm{i}}}{K_{\mathrm{m}}+x_{\mathrm{s}}^{\mathrm{i}}}
$$

where $V$ is the maximum reaction rate. Condition (14) is less restrictive than inequality (11) for lipid-faced sites and, owing to the large excess of water molecules, is always satisfied for aqueous-faced sites. Both the enzyme concentration $E$ and the maximum reaction rate $V$ depend linearly on the concentration $M$ of membrane lipid:

$$
\begin{array}{r}
V=k \cdot E=k \cdot\left(n_{\mathrm{E}} / N^{\mathrm{aq}}\right)=k \cdot\left[\left(n_{\mathrm{E}} / n_{\mathrm{M}}\right) /\left(n_{\mathrm{M}} / N^{\mathrm{aq}}\right)\right]= \\
k \cdot k^{\prime} \cdot M
\end{array}
$$

The proportion $n_{\mathrm{E}} / n_{\mathrm{M}}=k^{\prime}$, which is constant for any given membrane preparation, can be called the 'site density' (Parry et al., 1976).

It is practical interest to distinguish enzymic assay at (a) constant liposome concentration and (b) constant liposome/substrate concentration ratio. At constant liposome concentration the local concentration $x_{\mathrm{s}}^{1}$ to be entered into eqn. (15), is given by:

$$
\begin{gathered}
x_{\mathrm{s}}^{\mathrm{aq}}=S / F_{\mathrm{s}} \text { for an aqueous-faced site } \\
x_{\mathrm{s}}^{\mathrm{m}}=S \cdot P^{\mathrm{m}} / F_{\mathrm{s}} \text { for a lipid-faced site }
\end{gathered}
$$

yielding the rate expression:

$$
v=V \frac{S}{K_{\mathrm{m}}^{\mathrm{app}}+S}
$$

with

$$
K_{\mathrm{m}}^{\mathrm{app} .}=K_{\mathrm{m}}^{\prime} \cdot\left(1+P_{\mathrm{s}}^{\mathrm{m}} \cdot M+P_{\mathrm{s}}^{\mathrm{l}} \cdot L\right)
$$

and

$$
\begin{aligned}
& K_{\mathrm{m}}^{\prime}=K_{\mathrm{m}} \text { for an hydrophilic aqueous-faced site } \\
& K_{\mathrm{m}}^{\prime}=K_{\mathrm{m}} / P_{\mathrm{s}}^{\mathrm{m}} \text { for a lipid-faced site }
\end{aligned}
$$

The present treatment naturally leads to the definition of the new constant $K_{\mathrm{m}}^{\prime}$, which replaces the Michaelis constant $K_{\mathrm{m}}$ of single-phased enzyme systems. We propose to call $K_{\mathrm{m}}^{\prime}$ the 'effective Michaelis constant'. A plot of $M / v$, the inverse of the specific reaction rate, against $1 / S$ at constant liposomal concentration $(L)$ at various concentrations of membrane lipid $(M)$ :

$$
\frac{M}{v}=\frac{1}{k^{\prime} k}+\frac{K_{\mathrm{m}}^{\prime}\left(1+P_{\mathrm{s}}^{\mathrm{m}} \cdot M+P_{\mathrm{s}}^{1} \cdot L\right)}{k^{\prime} k} \cdot \frac{1}{S}
$$

mimics competitive inhibition by membrane lipid. Both

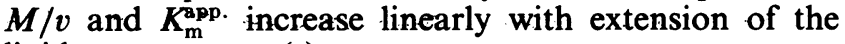
lipid compartment(s).

At constant substrate/liposome concentration ratio, substrate and liposomal lipid are introduced into the incubation mixtures at the fixed ratio $R=n_{\mathrm{S}} / n_{\mathrm{L}}$. Thus:

$$
S=n_{\mathrm{S}} / N^{\text {aq }}=\left(n_{\mathrm{S}} / n_{\mathrm{L}}\right) \cdot L=R \cdot L
$$

Substitution of $L=S / R$ into the distribution function (13) and application of the definition of $P_{\mathrm{s}}^{\mathrm{m}}$ yields:

$$
\begin{aligned}
x_{\mathrm{s}}^{\mathrm{aq}} & \left.=S / 1+P_{\mathrm{s}}^{\mathrm{m}} \cdot M+P_{\mathrm{s}}^{\mathrm{l}} \cdot S / R\right) \\
x_{\mathrm{s}}^{\mathrm{m}} & =S \cdot P_{\mathrm{s}}^{\mathrm{m}} /\left(\mathrm{I}+P_{\mathrm{s}}^{\mathrm{m}} \cdot M+P_{\mathrm{s}}^{\mathrm{d}} \cdot S / R\right)
\end{aligned}
$$

and the kinetic expression:

$$
v=V^{\mathrm{app}} \frac{S}{K_{\mathrm{m}}^{\mathrm{app}}}+S
$$

with

$$
\begin{aligned}
V^{\mathrm{app} .}= & V \frac{1}{1+K_{\mathrm{m}}^{\prime} \cdot P_{\mathrm{s}}^{\mathrm{l}} / R}=V \frac{R}{R+K_{\mathrm{m}}^{\prime} \cdot P_{\mathrm{s}}^{1}} \\
& K_{\mathrm{m}}^{\mathrm{app} .}=K_{\mathrm{m}}^{\prime} \frac{1+P_{\mathrm{s}}^{\mathrm{m}} \cdot M}{1+K_{\mathrm{m}}^{\prime} \cdot P_{\mathrm{s}}^{1} / R} \\
& \frac{K_{\mathrm{m}}^{\mathrm{app} .}}{V^{\text {app. }}}=\frac{K_{\mathrm{m}}^{\prime}}{V}+\frac{P_{\mathrm{s}}^{\mathrm{m}} \cdot K_{\mathrm{m}}^{\prime}}{V} \cdot M
\end{aligned}
$$

$K_{\mathrm{m}}^{\prime}$ equals $K_{\mathrm{m}}$ for hydrophilic aqueous-faced sites and $K_{\mathrm{m}} / P_{\mathrm{s}}^{\mathrm{m}}$ for lipid-faced sites.

Two additional types of binding site will now be considered. The binding of a homologous series of amphipathic compounds, such as fatty acids, to serum albumin can be conceived as a partition process on to a hydrophobic aqueous-faced binding site characterized by a dissociation constant $K^{\prime}$ (Tanford, 1980). $K^{\prime}$ can be represented as the ratio $K / P_{\mathrm{s}}^{\text {bs }}$ where $1 / K$ and $P_{\mathrm{s}}^{\text {bs }}$ take into account the energetic contributions of the hydrophilic head group and of the hydrophobic part of the fatty acids, respectively. Also, inhibition by benzene derivatives and $N^{1}$-alkylnicotinamide homologues of several water-soluble enzymes can be characterized by a similar constant $K_{\mathrm{i}} / P_{\mathrm{s}}^{\text {bs }}$ (Anderson et al., 1965; Royer \& Canady, 1968; Canady et al., 1976). Then, by analogy, binding to an aqueous-faced hydrophobic enzymic site can be described by:

$$
K_{\mathrm{m}}^{\prime}=K_{\mathrm{m}} / P_{\mathrm{s}}^{\mathrm{bs}}
$$

$P_{\mathrm{s}}^{\mathrm{bs}}$ will be called the 'incremental binding site (bs) partition coefficient'. Substitution of relationship (31) into eqns. (20) and (28)-(30) provides a description of the kinetic behaviour of hydrophobic aqueous-faced membrane-bound enzymic sites.

Conceivably, binding sites could partially overlap an aqueous compartment and a neighbouring phospholipid compartment (mixed binding sites). Studies of the action of membrane-bound neuraminidase on the lipophilic substrate disialoganglioside $\mathrm{G}_{\mathrm{D} 1 \mathrm{a}}$ and on the hydrophilic substrate sialyl-lactitol, in the presence of various hydrophilic and lipophilic degradation products of $G_{D 1 a}$, suggest that an aqueous-faced hydrophilic catalytic centre is located adjacent to the lipid phase of the membrane, the acyl chains of $G_{D 13}$ penetrating vertically into the phospholipid phase (Sandhoff \& Pallmann, 1978; Scheel et al., 1982). Active substrate thus would correspond to the properly positioned substrate molecules present in the membrane lipid. Introduction of the local concentration $x_{\mathbf{s}}^{\mathrm{m}}$ into eqn. (15) thus yields $K_{\mathrm{m}}^{\prime}=K_{\mathrm{m}} / P_{\mathrm{s}}^{\mathrm{m}}$ for a mixed binding site. Both hydrophobic aqueous-faced and mixed binding sites yield equations which are formally identical with those given by lipid-faced binding sites, but probably represent more realistic modes of binding.

Adaptation of the previous treatment to binding systems at equilibrium is straightforward. Consider the equilibrium reaction:

$$
\mathrm{E}^{\mathrm{i}}+\mathrm{S}^{\mathrm{i}} \rightleftharpoons(\mathrm{ES})^{\mathrm{i}} \quad x_{\mathrm{es}}^{\mathrm{i}} \cdot K=x_{\mathrm{e}}^{\mathrm{i}} \cdot x_{\mathrm{s}}^{\mathrm{i}}
$$

occurring in phase i. $K$ is a dissociation constant and $\mathrm{E}^{\mathrm{i}}, \mathrm{S}^{\mathrm{i}}$ and $(\mathrm{ES})^{\mathrm{i}}$ denote the receptor site, ligand and 
receptor-ligand complex, respectively. The relationship between the local concentrations of occupied $\left(x_{\mathrm{es}}^{\mathrm{i}}\right)$ and total receptor sites $\left(x_{\mathrm{es}}^{\mathrm{i}}+x_{\mathrm{e}}^{\mathrm{i}}\right)$ is given by:

$$
x_{\mathrm{es}}^{\mathrm{i}}=\left(x_{\mathrm{es}}^{\mathrm{i}}+x_{\mathrm{e}}^{\mathrm{i}}\right) \frac{x_{\mathrm{s}}^{\mathrm{i}}}{K+x_{\mathrm{s}}^{\mathrm{i}}}
$$

$x_{\mathrm{s}}^{\mathrm{i}}$ now is a true equilibrium concentration, thus rendering condition (14) and the assumption about the existence of a state of equilibrium, made for kinetic systems, redundant. Expressing the local concentrations in terms of total concentrations by using eqns. (17) and (18) one obtains a response equation at constant liposomal concentration $L$ which is formally identical with the kinetic expression (19):

$$
R_{\mathrm{b}}=R_{\infty} \frac{S}{K^{\mathrm{app}}+S}
$$

where $R_{\mathrm{b}}$ and $\boldsymbol{R}_{\infty}$ represent experimental responses proportional to the ligand concentration bound at equilibrium and at saturation, respectively. The expression for the apparent dissociation constant $K^{\text {app. }}$ is given by:

$$
K^{\mathrm{app} .}=K^{\prime} \cdot\left(1+P_{\mathrm{s}}^{\mathrm{m}} \cdot M+P_{\mathrm{s}}^{\mathrm{l}} \cdot L\right)
$$

where $K^{\prime}$ equals $K$ for hydrophilic aqueous-faced binding sites and $K / P_{\mathrm{s}}$ for the other types with $\boldsymbol{P}_{\mathrm{s}}$ equal to $P_{\mathrm{s}}^{\mathrm{m}}$ or $P_{\mathrm{s}}^{\text {bs }}$, as applicable. Similarly, for assays at constant ligand/liposome ratio one has:

$$
R_{\mathrm{b}}=R^{\mathrm{app}} \frac{S}{K^{\mathrm{app}}+S}
$$

where $R^{\mathrm{app} .}$ is an apparent maximum response parameter. Subsidiary expressions for $R^{\text {app. }}$ and $K^{\text {app. }}$ are the same as eqns. 28-30 with replacement of $V$ by $R_{\infty}$ and of $K_{\mathrm{m}}$ by $K$.

It will be clear that only kinetic coefficients such as $V$ and $K^{\prime}{ }_{m}$ and binding parameters such as $R_{\infty}$ and $K^{\prime}$ are adequate to characterize the corresponding membranebound enzymic or liganding systems. Omission of the required extrapolation procedures leads to ill-defined parameters and to considerable loss of information. It should also be realized that the effects of changes of the size of the lipid compartments $(M$ and $L$ ) on the response quantity $v\left(\right.$ or $R_{\mathrm{b}}$ ) and on the $K$ parameter $K_{\mathrm{m}}^{\text {app. }}$ (or $K^{\text {app. }}$ ) are fully accounted for by the distribution function $F_{\mathrm{s}}=$ $\left(1+P_{\mathrm{s}}^{\mathrm{m}} \cdot M+P_{\mathrm{s}}^{1} \cdot L\right)$ whereas the nature and facedness of the binding sites exclusively affect the effective constant $K_{\mathrm{m}}^{\prime}\left(\right.$ or $\left.K^{\prime}\right)$. A decrease in the specific reaction rate with extension of the membrane compartment has occasionally been taken to support a hydrophilic aqueousfaced site. Such conclusions are not warranted since, qualitatively, the dependence on the size of the lipid compartments is exactly the same for the other site types. Also, plots of a response quantity such as $1 / v$ against $1 / x_{\mathbf{s}}^{1}$, calculated on the basis of an assumed or experimental value of the partition coefficient and on postulating a given site type, have no diagnostic value because the local concentrations in the aqueous and lipid compartments vary in a constant proportion.

Frequently, kinetic or binding experiments performed with natural membranes or with proteoliposomes are performed in the absence of added liposomes. Such experiments can be modelled by omitting the liposome partition term $P_{\mathrm{s}}^{\mathrm{1}} \cdot L$ from the eqns. 17-23 pertaining to assays at constant liposome concentration. Extension of

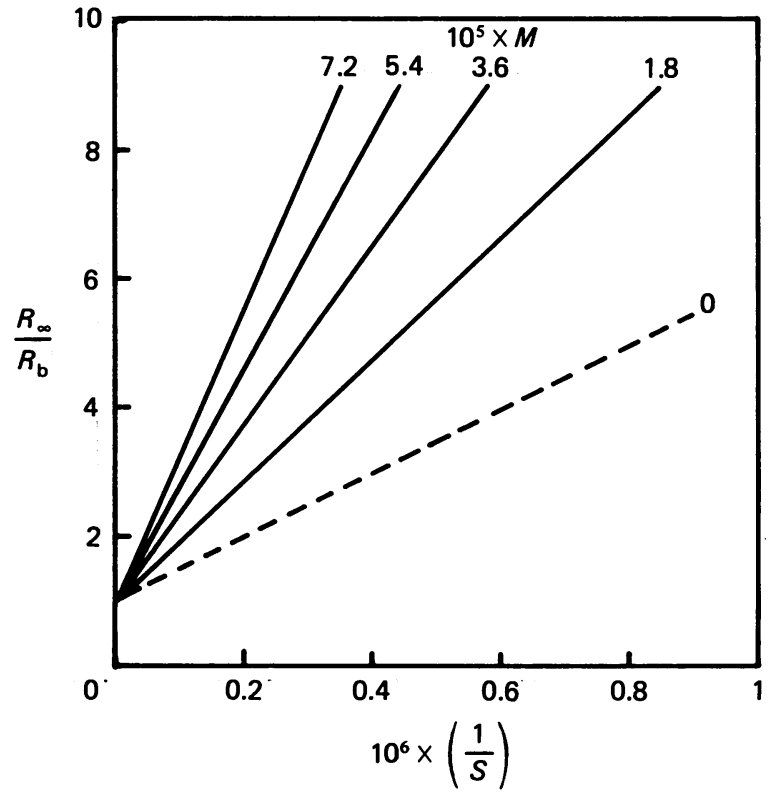

Fig. 1. Saturation curves of a membrane-bound receptor at varying membrane lipid concentration

Calculated double-inverse plots of the relative response $R_{\mathrm{b}} / \boldsymbol{R}_{\infty}$ against the total substrate concentration $S$ calculated with: $L=0 ; M=0,1.8 \times 10^{-5}, 3.6 \times 10^{-5}$, $5.4 \times 10^{-5}$ and $7.2 \times 10^{-5} ; P_{\mathrm{s}}^{\mathrm{m}}=5 \times 10^{4} ; K^{\prime}=5 \times 10^{-6}$. The plot mimics competitive inhibition by membrane lipid. Binding of cyclohexane to microsomal cytochrome $P-450$ conforms to this type of plot (Waterman et al., 1973).

the treatment to more complex systems could proceed as follows. For each phase, which is assumed to be homogeneous, any required kinetic and/or equilibrium relationship is formulated as a function of the local concentrations $x_{j}^{i}$. Reactions that are restricted to one of the phases are identified by the corresponding phase label, e.g. species intervening in binding of a ligand to added serum albumin should bear the label aq. Otherwise, the label $\mathrm{i}$ is used. Expressions $x_{\mathrm{j}}^{\mathrm{i}}=\mathrm{f}\left(F_{\mathrm{J}}, D\right)$, derived for each substrate, product, inhibitor etc. from the mass balance on $J$ and properly defined partition coefficients, are finally substituted into the set of kinetic and/or equilibrium equations.

\section{DISCUSSION}

In the present derivation of kinetic and equilibrium relationships for membrane-bound binding sites, mole fractions instead of molarities have been used. Apart from leading to more correct and easier interpretation of free energy relationships (Tanford, 1980), the use of mole fractions leads to more convenient description of multiphasic systems and also implies that volume fraction parameters become redundant. This is particularly important since definition of the volume of a lipid bilayer is somewhat arbitrary due to the fact that the accumulated solutes often are wholly (adsorption) or partially confined to the outside of the bilayers. Formulae for interconversion of partition coefficients defined on the commonly used molarity scale or on a mole fraction or molality basis have been given (Kamaya et al., 1981). 


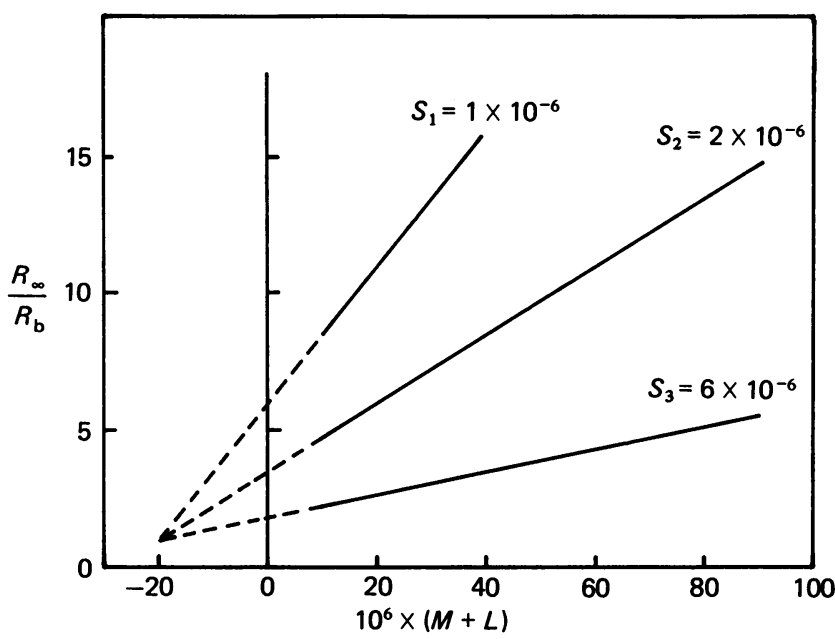

Fig. 2. Effect of extension of the lipid compartment by addition of liposomal lipid on $R_{\infty} / R_{\mathrm{b}}$ of a membrane-bound receptor

Curves were calculated for the total substrate concentration $S=1 \times 10^{-6}, 2 \times 10^{-6}$ and $6 \times 10^{-6}$, on the basis of the following data: $P_{\mathrm{s}}^{\mathrm{m}}=P_{\mathrm{s}}^{\mathrm{l}}=5 \times 10^{4} ; K^{\prime}=$ $5 \times 10^{-6} ; M=0.9 \times 10^{-5} ; L$ up to $81 \times 10^{-5}$. The abscissa value of the intersection $\left[1+P_{\mathrm{s}} \cdot(M+L)=0\right]$ yields the average partition coefficient $1 / P_{\mathrm{s}}=-(M+L)_{i}$. The predicted behaviour has been observed for the rotenoneinsensitive reduction of ubiquinone-1 (Ragan, 1978).

The present approach to modelling of membranebound kinetic and liganding systems relies heavily on the assumption of linear membrane partition of solutes. Transfer of amphipathic compounds such as bromosulphophthalein, oestrone sulphate, haem, bilirubin and pleuromutilin to phospholipid membranes has been described as non-linear saturable binding to phospholipid (Schuster et al., 1975; Tipping et al., $1979 b$ ). However, apparent 'saturation of binding sites' was only reached at ligand/phospholipid ratios as high as $0.1-0.35$. Also, in contrast to what one would expect for a basically non-linear process, transfer to phospholipid membranes was linear over considerable ranges of concentration for pleuromutilin (Schuster et al., 1975), neutral steroids, 2-acetamidofluorene and 4-dimethylaminoazobenzene (Tipping et al., 1979a). For the anionic amphipath bilirubin, constant partition coefficients were found for rough and smooth microsomal material up to bilirubin concentrations in the aqueous phase of $1.3 \mu \mathrm{M}$ (Meuwissen \& Heirwegh, 1982). At this concentration the intracellular carrier protein ligandin is already $98 \%$ saturated (affinity constant, $5 \times 10^{7} \mathrm{M}^{-1}$ ) but the ligand/phospholipid ratio is only about 0.01 . Similar limits to practically meaningful upper concentrations of ligand probably apply to many other amphipathic substances. The concept of binding to phospholipid completely fails to explain membrane accumulation of apolar substances such as cyclohexane (Tanford, 1980). Then, the simplest working hypothesis one may adopt is that, disregarding specific binding to membrane-bound receptors, linear partition adequately describes accumulation of amphipathic compounds into biological membranes (Parry et al., 1976). Apparent hyperbolic 'binding' at relatively high concentrations may be due to superimposition of a variety of phenomena.
Apart from a continuous change in the composition of the phospholipid solvent, eventually leading to considerable changes in the activity coefficients of the solutes in the membrane phase, oligomerization of amphipathic solutes is often important in the aqueous phase. The latter process may explain why the partition coefficients of chlorpromazine and methochlorpromazine in natural and synthetic bilayer membranes, at fixed membrane concentration, decreased with increasing solute concentration, but were constant when the membrane concentration was varied and the solute held constant at low concentration (Welti et al., 1984). Electrostatic effects, which will decrease partition of charged compounds (Haynes \& Staerk, 1974), can be diminished by maintaining the ionic strength at a rather high value, e.g. 0.1 M (McDaniel \& McLaughlin, 1985). High ionic strengths will also inhibit unequal distribution of charged solutes over the lumenal and surrounding aqueous phases.

As a consequence of the linear-partition hypothesis the saturation curves are formally identical with those curves obtained for similar single-phase systems. For example, the presently discussed systems yield rectangular hyperbolic curves (Fig. 1). However, the kinetic and equilibrium coefficents obtained are generally functions of the partition coefficients and the lipid concentration. Meaningful assessment of such systems requires extrapolation to zero lipid concentration. Extension of the lipid compartment affects the response variable and some parameters of the systems, e.g. by causing linear increases of $K_{\mathrm{m}}^{\mathrm{app}}$. and of $M / v$ with added phospholipid (Fig. 2).

The derived models permit determination of the membrane partition coefficients and certain kinetic coefficients or binding parameters from appropriate experimental data. It is of practical interest that the required total concentrations $X=n_{\mathrm{x}} / N^{\mathrm{aq}}(\mathrm{X}=\mathrm{S}, \mathrm{P}, \mathrm{M}$ etc.) can, to a sufficient approximation, be replaced by the numerical value of the ratio (molarity of $\mathrm{X}) / 55.49$, since the systems are generally quite dilute $\left(n_{\mathrm{x}} \ll 0.1 \mathrm{~mol}\right)$. Treatment of kinetic or binding data, obtained at constant concentration of liposomal and membrane lipid, is outlined in Fig. 3. Each saturation curve yields a maximum response parameter, $V$ or $R_{\infty}$, and an apparent kinetic $\left(K_{\mathrm{m}}^{\mathrm{app}}\right)$ or equilibrium constant $\left(K^{\text {app. }}\right)$. By a double extrapolation procedure one further obtains from the values of the apparent $K$ parameter, the effective parameter $K^{\prime}$ and partition coefficients $P_{\mathrm{s}}^{\mathrm{m}}$ and $P_{\mathrm{s}}^{\mathbf{l}}$. Assays at a constant substrate/liposome ratio require more complicated extrapolation procedures (eqns. 28-30) but offer the advantage that the substrate solutions needed to establish a saturation curve can be obtained by diluting a single liposome/substrate mixture.

Obviously, the underlying assumption of bulk-phase partition into an isotropic fluid can only lead to an approximate description of partition into phospholipid bilayers, which rather should be seen as quasibidimensional highly organized fluids (Simon et al., 1977, 1979). For example, partition into such membranes shares, with stereospecific binding to the surface of a binding pocket, the imposition of restraints on the orientation and position of the ligands. These may either be located superficially or penetrate the bilayers to various depths depending on the relative polarity of the sub-layers of the membranes (Simon et al., 1977) and on the structure of the solutes (Jain \& Wu, 1977; Kamaya 

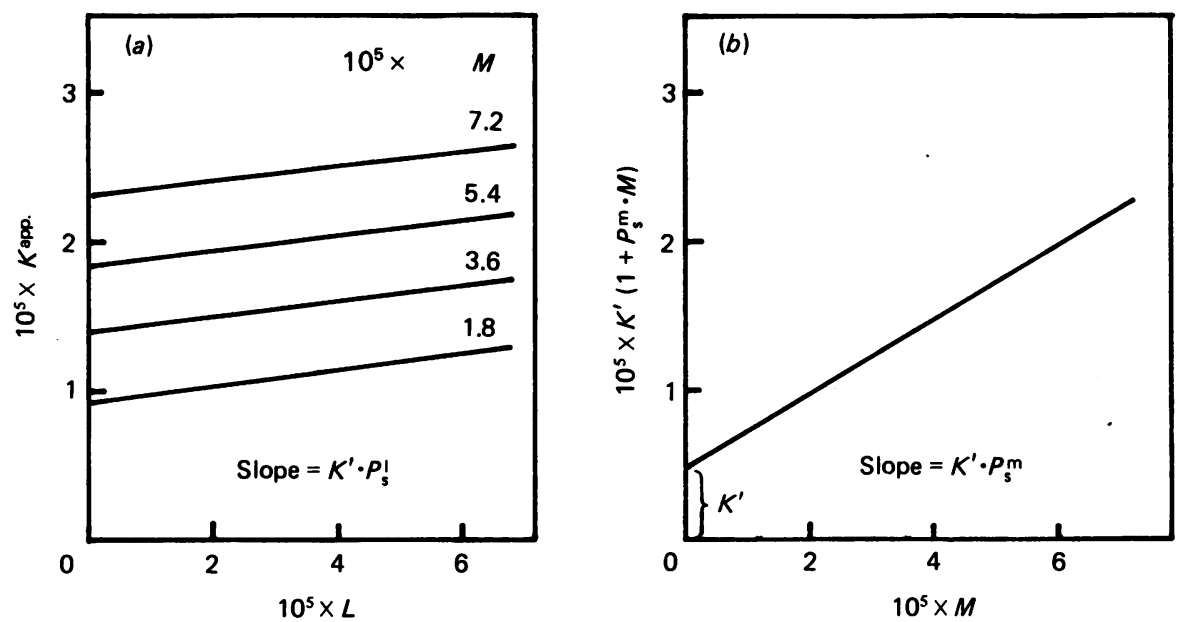

Fig. 3. Treatment of binding data obtained at constant liposomal concentration (eqns. 17-22, 34, 35): double extrapolation of $K^{\text {app. }}$ to obtain $R_{\infty}$, the effective dissociation constant $K^{\prime}$, and the partition coefficients $P_{s}^{m}$ and $P_{s}^{\prime}$

The calculations were based on the following data: $K^{\prime}=5 \times 10^{-6} ; P_{\mathrm{s}}^{\mathrm{m}}=5 \times 10^{4} ; P_{\mathrm{s}}^{\mathrm{l}}=1 \times 10^{4} ; M$ and $L, 1.8 \times 10^{-5}, 3.6 \times 10^{-5}$, $5.4 \times 10^{-5}$ and $7.2 \times 10^{-5} ; S$ values correspond to the range $0.1-0.7$ for $R_{0} / R_{\infty}$. By fitting the rectangular hyperbola (eqn. 36) to $\left(S_{1}, R_{\mathrm{b}, \mathrm{j}}\right)$ data pairs, obtained at constant membrane $(M)$ and liposome concentration $(L)$, one obtains the maximal response

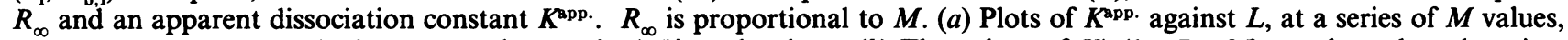
yield $K^{\prime} \cdot\left(1+P_{\mathrm{s}}^{\mathrm{m}} \cdot M\right)$ as the intercept values and $K^{\prime} \cdot P_{\mathrm{s}}^{\mathrm{l}}$ as the slope. $(b)$ The values of $K^{\prime} \cdot\left(1+P_{\mathrm{s}}^{\mathrm{m}} \cdot M\right)$ are then plotted against $M$, yielding $K^{\prime} \cdot P_{\mathrm{s}}^{\mathrm{s}}$ and $K^{\prime}$. The predicted linear dependence of $K^{\mathrm{spp} .}$ on $M$ has been observed for the binding of several homologues of benzene and barbiturate to microsomal cytochrome P-450 (Backes et al., 1982, 1984) and for microsomal hydroxylation of benzo[a]pyrene (Nebert \& Gelboin, 1968). Treatment of systems devoid of liposomal carrier material $(L=0)$ is considerably more simple since $K^{\mathrm{app} .}=K^{\prime} \cdot\left(1+P_{\mathrm{s}}^{\mathrm{m}} \cdot M\right)$. A single extrapolation as shown in panel $b$ thus, is sufficient.
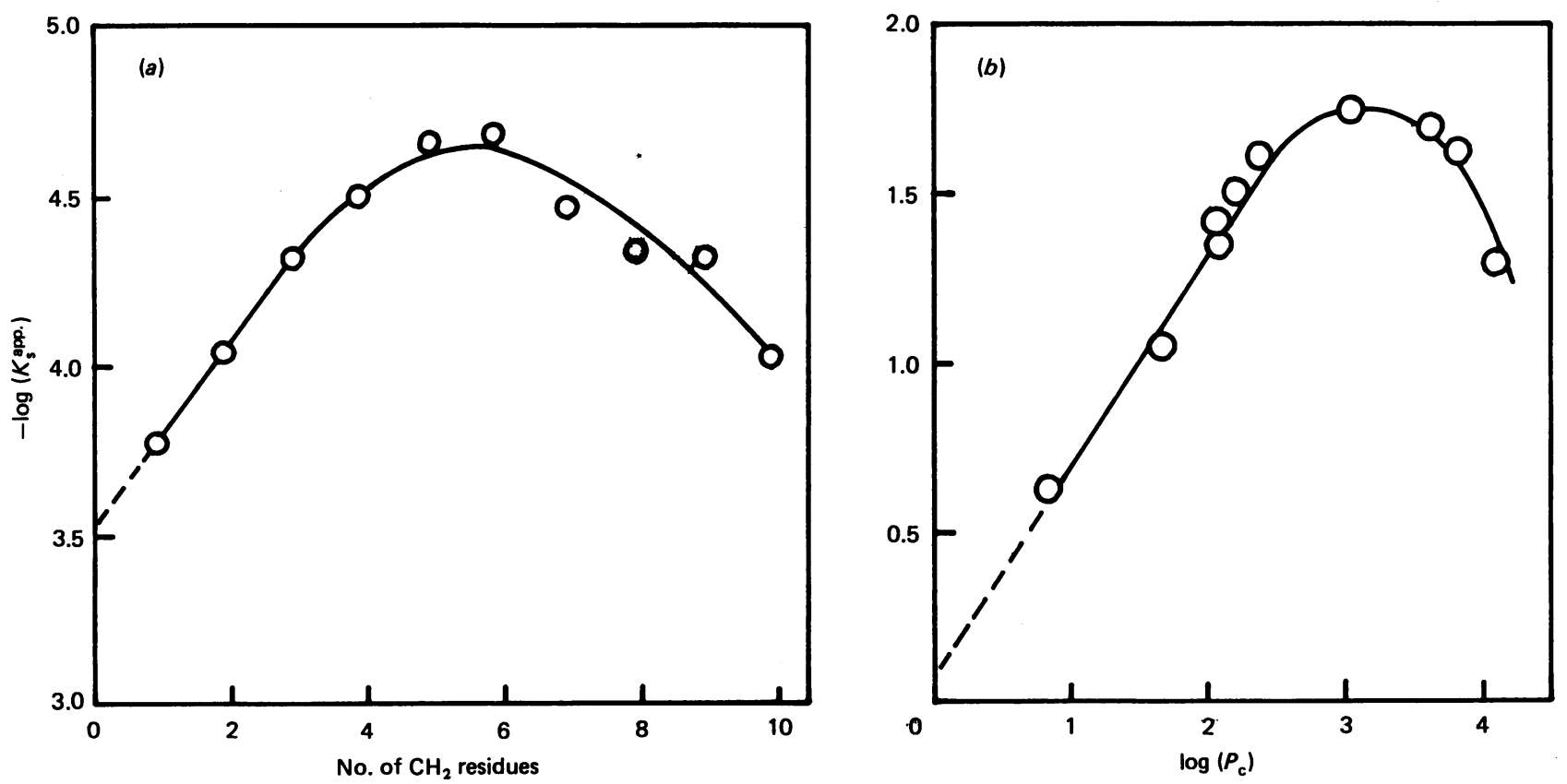

Fig. 4. Dependence of the apparent spectral dissociation constant $K_{s}^{\text {app. }}$ of microsomal cytochrome $P-450$ on membrane partition of homologous ligands

Values of $-\log \left(K_{\mathrm{s}}^{\text {app. }}\right)$ for $(a)$ 4-alkyl-substituted pyridines (Born \& Hadley, 1980) and (b) 5-ethyl-5-alkyl-substituted barbiturates (Yih \& van Rossum, 1977) are plotted against the number of $\mathrm{CH}_{2}$ residues and $\log \left(\boldsymbol{P}_{\mathrm{c}}\right)$ (octanol/water system), respectively. Both $K_{\mathrm{s}}^{\mathrm{app} .}$ and $P_{\mathrm{c}}$ are in molarity units. The linear increase at relatively small molecule masses is compatible with a lipid-faced, a hydrophobic aqueous-faced or a mixed binding site. Deviation form linearity at relatively high molecular masses may be due to sterically hindered binding to a physically circumscribed binding pocket. As indicated by work of Backes et al. (1982, 1984), the values of $\mathrm{d}\left(\log P_{\mathrm{b}}^{\mathrm{m}}\right) / \mathrm{d} n_{\mathrm{CH}_{3}}$ and $\mathrm{d}\left(\log K^{\prime}\right) / \mathrm{d} n_{\mathrm{CH}_{2}}$ are sensitive indicators of the nature of the hydrophobic environments of the bilayer membrane and of the binding site, respectively. 
et al., 1981). The experimental membrane partition coefficients thus are macroscopic parameters that, in general, represent averages of partition coefficients applicable to the various anisotropic sub-layers of the membranes.

Without some independently obtained information about the nature and facedness of the binding site one cannot know whether an experimental $K^{\prime}$ value needs to be corrected for partition. The problem can be solved partially by assaying a number of compounds with the same catalytically sensitive or liganding group and variable substituents conferring sufficiently different membrane partition (Fig. 4). For example, for a hydrophilic aqueous-faced catalytic site $K_{\mathrm{m}}^{\prime}$ is expected to correspond to the true $K_{\mathrm{m}}$ value since, by hypothesis, such a site is devoid of hydrophobic binding patches. Although such sites undoubtedly have important functions in metabolism, it is likely that amphipathic compounds instead interact with one or the other type of hydrophobic binding site. For a wide variety of structurally related or homologous compounds kinetic assays of substrate conversion $\left(K_{\mathrm{m}}^{\text {app. }}\right)$ by and inhibition $\left(K_{1}^{\text {app. }}\right)$ of microsomal enzymes, and binding assays of microsomal cytochrome $P-450\left(K_{\mathrm{s}}^{\text {app }}\right)$ indicate that, in general, $-\log \left(K^{\text {app. }}\right)$ increases linearly with the logarithm of the partition coefficient for several low-molecularmass members of each series (see, e.g. Martin \& Hansch, 1971; Yih \& van Rossum, 1977; Sípal et al., 1979; Born \& Hadley, 1980; Backes et al., 1982, 1984) (Fig. 4). Most of these studies pertain to cytochrome $P-450$. The observed dependence on partition rules out an aqueous-faced fully hydrophilic binding site for cytochrome $P-450$ but leaves a choice between lipid-faced, hydrophobic aqueous-faced and mixed sites. It is difficult to imagine this site to be lipid-faced. If so, the site would be situated at a fixed depth, whereas the widely variable polarities of the specific part and of the variable hydrophobic parts of the substrates would rarely result in positioning the sensitive group exactly opposite to the active site. Clearly, an aqueous-faced site with hydrophobic binding patches provides greater flexibility than a lipid-faced site. For homologues of benzene and of barbiturate the decreases in free energy per methylene group for binding to microsomal cytochrome $P-450$ (calculated from $K_{\mathrm{s}}^{\prime}$ ) and for transfer to the bilayer lipid (calculated from $P_{\mathrm{s}}^{\mathrm{m}}$ ) were significantly different (Backes et al., 1982, 1984), confirming the notion that the binding site differs from a lipid-faced site. For bulk-phase systems, $\log \left(P_{\mathrm{s}}^{\mathrm{m}}\right)$ is expected to increase linearly with surface area or, for a homologous series, with the length of the alkyl chain (Tanford, 1980; Backes et al., 1982, 1984). Deviation from this behaviour for the high-molecular-mass members of homologous series of ligands (Fig. 4) may signal special features such as steric hindrance at the surface of a binding pocket, gradual reorientation with increasing alkyl chain length or pronounced disturbance of the bilayer.

The concept of membrane partition appears to be applicable to many types of small molecules, leads to simple expressions for membrane-related response variables and, of course, affords a much better description of membrane systems than when they are treated as if they were single-phased. However, the treatment offered is limited in two respects. First, the partialequilibrium assumption made in deriving the kinetic relationships may not apply to the kinetics of some membrane-bound enzymes, but seems a reasonable first approximation in the absence of evidence to the contrary. In this respect, changes of enzyme activity by agents that alter membrane fluidity (Dipple \& Houslay, 1978; Scheel et al., 1982; Storch \& Schachter, 1984) may be of interest. For example, an increase of neuraminidase activity accompanying increasing membrane fluidity has been related experimentally to diffusion-limited lateral transport of substrate to a mixed-type enzymic site (Sandhoff \& Pallmann, 1978). Furthermore, octanol/ water or similar bulk-phase partition coefficients have intensively been used to interpret membrane-related phenomena observed in vitro or in vivo (Martin \& Iansch, 1971; Hansch \& Dunn, 1972). Although membrane partition coefficients, derived on the bulkphase assumption, reflect the average polarity of phospholipid bilayers (Backes et al., 1982, 1984), it will be necessary to take into account the consequences of treating partition into a quasi-bidimensional phase as if an isotropic bulk phase were involved.

We thank the Fund for Medical Scientific Research of Belgium for financial support. J.A.T.P.M. is a Research Associate for the National Fund for Scientific Research of Belgium.

\section{REFERENCES}

Anderson, B. M., Reynolds, M. J. \& Anderson, C. D. (1965) Biochim. Biophys. Acta 99, 46-55

Backes, W. L., Hogaboom, M. \& Canady, W. J. (1982) J. Biol. Chem. 257, 4063-4070

Backes, W. L., Means, M. \& Canady, W. J. (1984) J. Biol. Chem. 259, 10092-10099

Bartlett, K., Bartlett, P., Bartlett, N. \& Sherratt, H. S. A. (1985) Biochem. J. 229, 559-560

Born, J. L. \& Hadley, W. M. (1980) Proc. West. Pharmacol. Soc. 23, 259-262

Canady, W. J., Westfall, S., Wirtz, G. H. \& Robinson, D. A. (1976) Immunochemistry 13, 229-233

Cho, A. K. \& Miwa, G. T. (1973) Drug. Metab. Dispos. 2, 477-483

Dipple, I. \& Houslay, M. D. (1978) Biochem. J. 174, 179-190

Hansch, C. \& Dunn, W. J., III (1972) J. Pharm. Sci. 61, 1-18

Haynes, D. H. \& Staerk, H. (1974) J. Membr. Biol. 17, 313-340

Heirwegh, K. P. M., Campbell, M. \& Meuwissen, J. A. T. P. (1978) in Conjugation Reactions in Drug Biotransformation (Aitio, A., ed.), pp. 191-202, Elsevier/North-Holland Biomedical Press, Amsterdam, Oxford and New York

Hill, M. W. (1974) Biochim. Biophys. Acta 356, 117-124

Hook, G. E. R., Bend, J. R. \& Fouts, J. R. (1973) Chem.-Biol. Interact. 7, 205-222

Jain, M. K. \& Wu, N. W. (1977) J. Membr. Biol. 34, 157-201

Kamaya, H., Kaneshina, S. \& Ueda, I. (1981) Biochim. Biophys. Acta 646, 135-142

Martin, Y. C. \& Hansch, C. (1971) J. Med. Pharm. Chem. 14, 777-779

McDaniel, R. \& McLaughlin, S. (1985) Biochim. Biophys. Acta 819, 153-160

Meuwissen, J. A. T. P. \& Heirwegh, K. P. M. (1982) in Bilirubin, vol. 2, Metabolism (Heirwegh, K. P. M. \& Brown, S. B., eds.), pp. 39-83, CRC Press Inc., Boca Raton, Florida, U.S.A.

Nebert, D. W. \& Gelboin, H. V. (1968) J. Biol. Chem. 243, 6242-6249

Noy, N. \& Zakim, D. (1985) Biochemistry 24, 3521-3525 
Parry, G., Palmer, D. N. \& Williams, D. J. (1976) FEBS Lett. 67, 123-129

Ragan, C. I. (1978) Biochem. J. 172, 539-547

Royer, G. \& Canady, W. J. (1968) Arch. Biochem. Biophys. 124, 530-534

Sandhoff, K. \& Pallmann, B. (1978) Proc. Natl. Acad. Sci. U.S.A. 75, 122-126

Scheel, G., Acevedo, E., Conzelmann, E., Nehrkorn, H. \& Sandhoff, K. (1982) Eur. J. Biochem. 127, 245-253

Schuster, I., Fleschurz, C. \& Helm, I. (1975) Eur. J. Biochem. 51, 511-519

Simon, S. A., Stone, W. L. \& Busto-Latorre, P. (1977) Biochim. Biophys. Acta 468, 378-388

Simon, S. A., Stone, W. L. \& Bennett, P. B. (1979) Biochim. Biophys. Acta 550, 38-47

Sípal, Z., Anzenbacher, P., Putz, Z., Chlumský, J. \& Krivanová, M. (1979) Acta Biol. Med. Ger. 38, 483-494
Storch, J. \& Schachter, D. (1984) Biochemistry 23, 11651170

Tanford, C. (1980) The Hydrophobic Effect, 2nd edn., pp. 5 and 157, John Wiley and Sons, New York, Chichester, Brisbane and Toronto

Tipping, E., Ketterer, B. \& Christodoulides, L. (1979a) Biochem. J. 180, 319-326

Tipping, E., Ketterer, B. \& Christodoulides, L. (1979b) Biochem. J. 180, 327-337

Waterman, M. R., Ullrich, V. \& Estabrook, R. W. (1973) Arch. Biochem. Biophys. 155, 355-360

Welti, R., Mulliken, L. J., Yoshimura, T. \& Helmkamp, G. M., Jr. (1984) Biochemistry 23, 6086-6091

Whitmer, D. I., Russell, P. E., Ziurys, J. C. \& Gollan, J. L. (1986) J. Biol. Chem. 261, 7170-7177

Yin, T. D. \& van Rossum, J. M. (1977) Biochem. Pharmacol. 26, 2117-2120

Received 14 September 1987/14 January 1988; accepted 29 April 1988 